\title{
Evaluation and selection of information technology based on simulation
}

\author{
ZHI Li-fu
}

Department of Electric and Electronic Engineering; Shijiazhuang Vocational Technology Institute;

Shijiazhuang; Hebei 050081; China

Keywords: organizational learning; explore learning; exploitation learning; information technology

\begin{abstract}
By introducing the time value of knowledge and natural selection pressures, extend the existing organizations to explore and multi-body simulation model uses learning and assess the impact of the two types of IT organizational performance. One technique is to support mutual learning between the lateral members of the organization, and the other is to support organizations and members learn from each other longitudinally technology. Simulation results show that the external environment is complex, volatile, high-value high-level knowledge of the marginal members of learning ability, small size and staff turnover, competitive pressures faced by small organizations, transverse technology is more favorable; otherwise the longitudinal technology is more favorable. Organizations should be based on the external environment on the exploration and use of requirements, and to explore and exploit the nature of internal environment, there is the tendency to combine different information technologies to enable organizations to better adapt to the environment.
\end{abstract}

\section{Introduction}

Organization's ability to acquire and manage knowledge of becoming one of the key factors affecting the performance of the organization, and information technology as a carrier and transmission of knowledge, as a means to enhance the ability of many organizations. However, the existing simulation study ignores the time value of knowledge and natural selection pressures, underestimate the advantages of longitudinal technology. Therefore, information technology more comprehensive and more accurate assessment of the choice, the time value of knowledge as well as the need to "discount" into organizational performance metrics, to explore how technology and vertical transverse technologies affect the organization performance. In this paper, the time value of knowledge and "discount" into account, in order to organize the utility of different information technologies to replace existing models of knowledge as a measure of organizational performance, and on this basis, for March and extends the model of Miller et al. experiment and analysis.

\section{Adaptation of information technology and organization}

\section{IT and organizational learning}

Organizational learning is an experience and knowledge in the middle of the process, from interactive experience and the environment. Organizational learning includes two forms: Exploration and Utilization. Explore means to maintain the diversity of knowledge, the discovery of new knowledge and organizational memory replaces the existing content, and the use of means of diffusion of existing knowledge, refining, reuse, typically reduces intellectual diversity, to reach an agreement. Organizations need to balance the relationship between the two in order to adapt to the changing environment.

This paper is focused on two types of IT: Portrait of technical support and organizational members learn from each other, learn from each other between the lateral support members. Most existing studies agree longitudinal technology can rapidly improve the level of knowledge, but knowledge converge fast, long-term performance is poor, with the use of nature, reflect only short-term advantage; transverse technology can save the diversity of knowledge, exploratory nature, long reach a higher level of knowledge. This seems to deny the longitudinal technical and organizational managers existence. However, such a conclusion is mainly because the existing 
research organizations as a measure of the level of knowledge objects, ignoring the knowledge of the value created can accumulate and magnify over time, and because of uncertainty about the future of people and more attention to the recent value. But these factors are essential for the development and survival of the organization, when making IT decisions need to be taken into account.

\section{Time value and natural selection pressures knowledge}

Knowledge is organized source of value creation. Organization by integrating different sources of knowledge activities, and make it to the intrinsic value outside of value creation. But only in order to create value for the dynamic use of knowledge. Therefore, knowledge to create value depends on two aspects: the integration of available resources and dynamic application of knowledge, the matching degree of knowledge and the external environment, as reflected in the use of models to explore the level of knowledge. However, it has to create value can rejoin the organization as an additional resource for the re-creation. This makes knowledge have the time value of: the recent level of knowledge to improve the value created greater than the long-term knowledge of the same degree. Existing models consider only the dynamic use of knowledge, while ignoring the growing role of knowledge resources, not comprehensive.

On the other hand, even if the different IT enables organizations to achieve the same performance, it may not be able to obtain the same level of satisfaction. The reason is that organizations face varying degrees of natural selection pressure on the value of the distribution over time have different requirements, so there are different "discount rate." "Discount rate" refers to the relatively recent value when people on long-term value play "discount rate" reflect the pressure of natural selection. Organizations face greater pressure of natural selection, the more rapidly the requirements to benefit and reduce risk, and therefore have a higher discount rate, the lower the effectiveness of long-term value; the other hand, relatively high long-term value of the utility. Existing unstudied different requirements of different organizations. Therefore, value creation requires a combination of knowledge of the different requirements of the process and the organization, incorporated in the existing knowledge of the time value of the model and the "discount" to the organization of utility IT organization performance as the evaluation criteria in order to be more IT comprehensively and accurately evaluate and choose.

\section{Model}

To study the effectiveness of different types of tissue technology portfolio, this paper extends the model of March and Miller et al. The existing model was modified and extended to help compare and validate existing conclusions strengthen the accumulation of existing understanding of the theory and knowledge.

\section{Existing model feature}

Firstly, the adoption of the four main characteristics March model:

(1). There is an objective external environment, expressed as M-dimensional vector, for each dimension value of 1 or -1 . $M$ represents the environmental complexity. Within each cycle, each dimension of probability to the opposite value of $\mathrm{N}, \mathrm{N}$ representative of the degree of environmental disturbance.

(2). The organization and its members have knowledge of the external environment, expressed as M-dimensional vector. Each dimension is 1, -1 or 0 , corresponding to the dimensions of the external environment on behalf of the faith. 0 indicates lack of knowledge; otherwise, a dimension equal to the external environment (not equal) that hold the right (wrong) belief on that dimension. Members of the knowledge in the proportion of $U 0$ reflect a level field where strange.

(3). Within each cycle, each member has a probability of $\mathrm{T}$ is replaced by a new member. $\mathrm{T}$ on behalf of staff turnover. Second, Miller and other learning to expand by increasing the lateral March's model, we use it as a fifth feature:

(4). Among members of the organization can learn from each other. This process is the process of applying lateral technology. Members of each organization to identify neighbors in the highest level of knowledge and higher than its own members, to replicate for their own faith convictions. Miller 
and other members will be placed on the regular networks, each member has four neighbors. Miller also discussed and other small-world effect is more realistic, the results show that small-world effect and vertical learning process has an alternative. This article focuses on the combined effect of longitudinal and transverse technology, in order to avoid the effects of network structure and longitudinal technology to produce confusion, this article ignores the small-world effect.

In addition, this paper argues in favor of conviction right value creation, erroneous beliefs will cause damage. Therefore, it also adopted the Miller and other ways to measure the level of knowledge: the difference between right and wrong belief that the proportion of the total dimension of $\mathrm{M}$ measures the level of knowledge.

\section{New features}

Members acquire knowledge, it is often difficult to distinguish between right and wrong for each dimension of faith, which makes learning organization members exhibit randomness. Therefore, increasing the sixth feature:

(5). Learning organization members are random and incomplete. As members to the level of knowledge than their neighbors or organizational learning, the random selection portion dimension of faith, with the probability of L will copy it for their own beliefs. L behalf of the members ability to learn. Dynamic changes in the environment so that knowledge of timeliness, new knowledge is more accurate. Therefore, when organizations update their knowledge, members will learn to organize as soon as possible. At the same time, has the attention of the members is limited, at the same time individuals can not learn the different sources of knowledge. Accordingly, increasing the seventh feature article:

(6). Transverse and longitudinal technology application technology has an alternative. In each cycle, the application of probability R horizontal technical study, with probability 1-R technology applied longitudinal study. The application of horizontal technical, members of the study to a higher level of knowledge of the neighbor; the application of longitudinal technology, organizational learning to the members first, then a member of the organization to learn.

(7). It is a function of organizational knowledge and knowledge of the members of the average level of the value created by the interaction of knowledge, and accumulate over time. This article assumes that the i-th cycle of the new to create value for:

$$
\Delta O_{i}=O_{i-1} \times\left(O K_{i} \times A K_{i}\right)^{K}
$$

According to the analysis above, the effectiveness of IT organizations rely on IT to create value and natural selection pressures faced by the organization. Therefore, the organization will be "discounted" to calculate some IT organizations choose a combination of utility and information technology as a basis for assessing the performance according to their "discount rate" for future value. Accordingly, this article increases eighth feature:

(8). Based on the performance of different organizations to assess the effectiveness of information technology. In order to facilitate the calculation, this article will forward as a standard for comparison, is about the value of different periods are in accordance with the "discount rate" to calculate the long-term effectiveness. Therefore, the organization of the cumulative value of the i-th utility as follows:

$$
\operatorname{Utility}\left(O_{i}\right)=\left(O_{i-1}+\Delta O_{i}\right) \times(1+D)
$$

In each simulation cycle, the first computing organizations and members of the knowledge and the knowledge of the value and utility, then randomly select applications or longitudinal horizontal technical basis for technical parameters $\mathrm{R}$, and finally set the environment disturbance and movement of people. Role models as well as the characteristics of the process as shown in Fig1. 


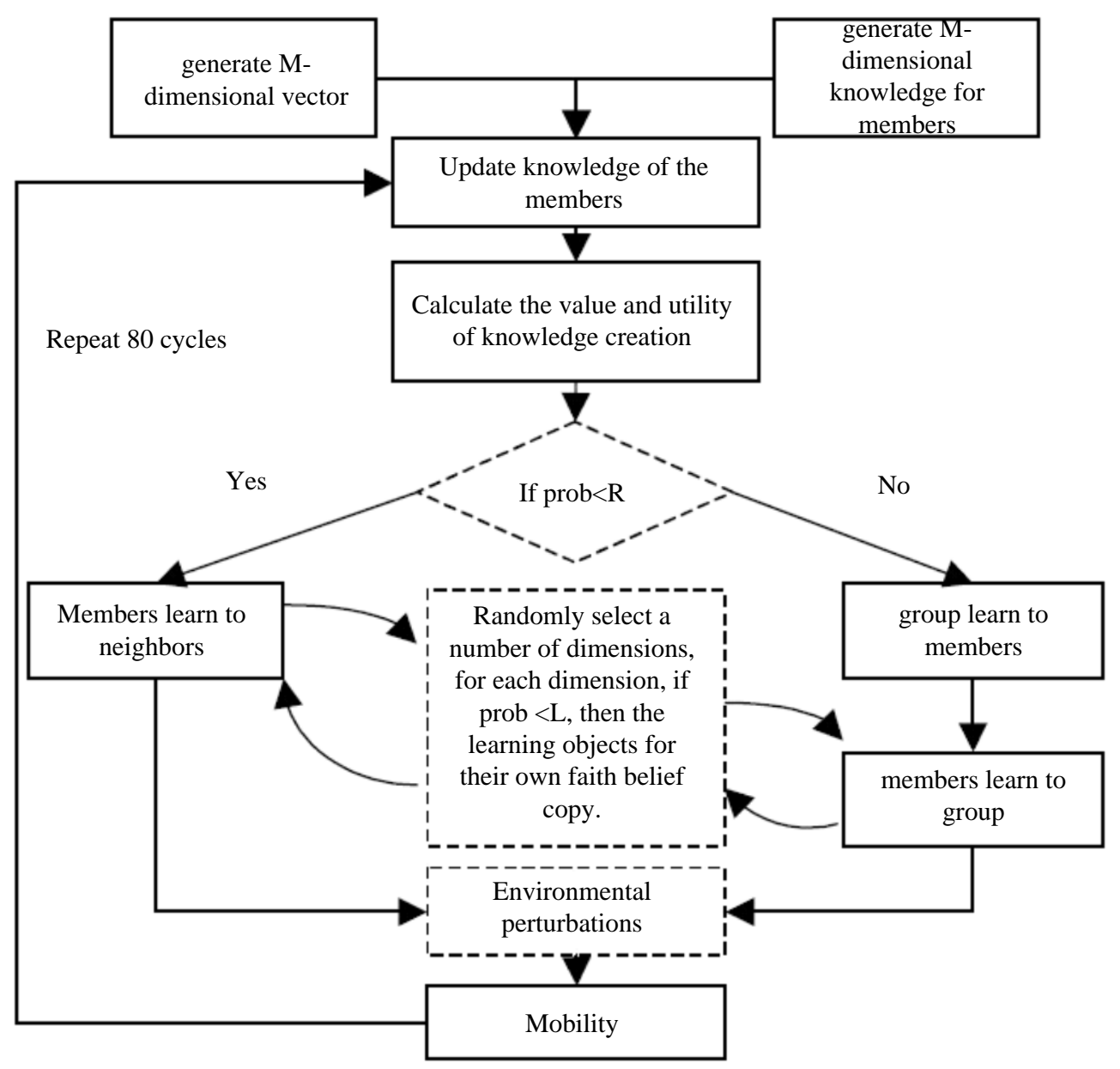

Figure 1. Model flowchart

\section{Simulation results}

As used herein, Netlogo5.0 software simulation, and using a different random number seed setting run 100 times for each parameter. In this paper, the average results of 100 experiments as the basis for analysis, in order to avoid the effects of randomness. In addition, it also conducted a sensitivity analysis to verify the stability of the conclusion. The experimental parameters and sensitivity analysis are shown in Table 1 set. The number of simulation results depends on the specific set of parameters; it has no practical significance, so this article will focus on parameter changes caused by changes in outcomes.

Table 1. Parameter setting and sensitivity analysis

\begin{tabular}{|c|c|c|c|}
\hline Parameters & Meaning & Parameters setting & Sensitivity analysis \\
\hline $\mathrm{P}$ & Organization size & $50,100,15$ & $25,125,200$ \\
\hline M & $\begin{array}{l}\text { Environmental } \\
\text { Complexity }\end{array}$ & $30,90,15$ & 60,120 \\
\hline U & Strange field level & $0.1,0.3,0.5,0.7,0$ & $0.2,0.4,0.6,0.8$ \\
\hline $\mathrm{L}$ & $\begin{array}{c}\text { Individual learning } \\
\text { ability }\end{array}$ & $0.1,0.3,0.5,0.7,0.9$ & $0.2,0.4,0.6,0.8,1$ \\
\hline $\mathrm{T}$ & Staff turnover & $0,0.1,0.2,0.3$ & $0.05,0.15,0.25,0.4$ \\
\hline $\mathrm{N}$ & perturbations rate & $0,0.01,0.03,0.05$ & $0.02,0.07,0.1$ \\
\hline $\mathrm{D}$ & selection pressure & $0,0.01,0.02$ & $0.005,0.05$ \\
\hline K & $\begin{array}{c}\text { Coefficient of } \\
\text { knowledge value }\end{array}$ & 1,3 & 3,5 \\
\hline $\mathrm{R}$ & $\begin{array}{l}\text { horizontal technical } \\
\text { applications }\end{array}$ & $0.1,0.3,0.5,0.7,0.9$ & $0,0.2,0.4,0.6,0.8$ \\
\hline
\end{tabular}


The results showed that the level of knowledge from long-term perspective, the conclusions and broadly in line with previous studies in this paper: horizontal and vertical, respectively, showing the nature of technology exploration and utilization of knowledge to enhance the former makes slow, but long-term to achieve a higher level ; the latter so quickly enhance the level of knowledge, but knowledge lead to long-term convergence too difficult to achieve a high level. This conclusion is in high staff turnover Exception: horizontal technical loss of ability to explore, weakening it in the short and long-term performance.

From the organization's effectiveness perspective, it is concluded that a more complex conclusion. In this paper, the level of each parameter of $\mathrm{R}$ were ANOVA and SNK multiple comparison, concerns changes in the ranking of different lateral and longitudinal technology portfolio parameters caused by changes (significance level of 0.01, not significant portfolio of technologies considered as ranking tied) .

Figure 2 shows the eight internal and external environmental parameters and R interaction. SNK multiple comparison below with the results of a variety of techniques with different combinations of performance parameters were analyzed.

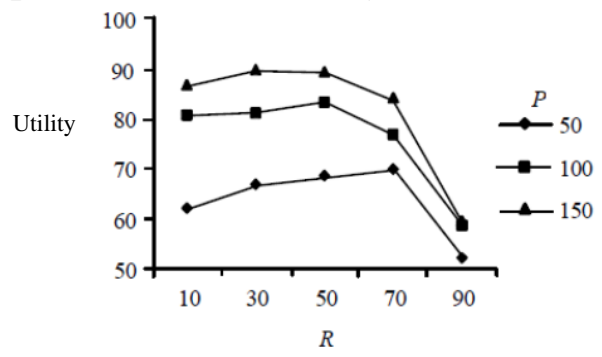

(a)

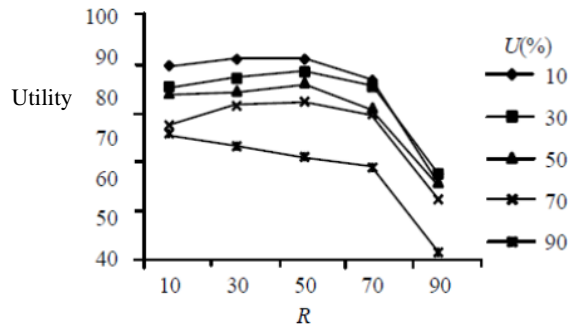

(c)

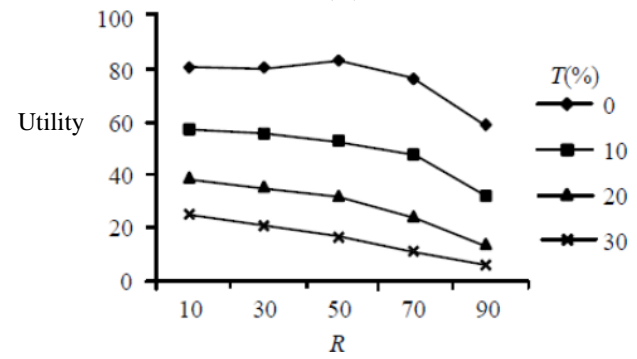

(e)

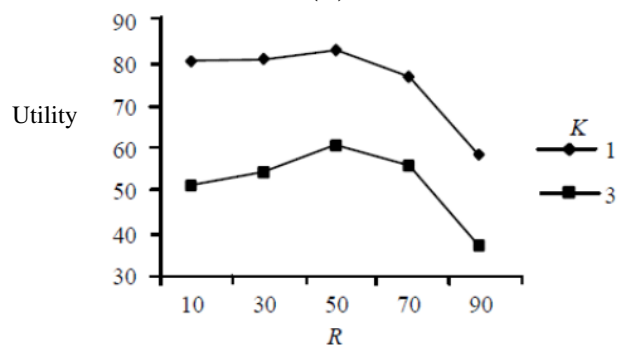

(g)

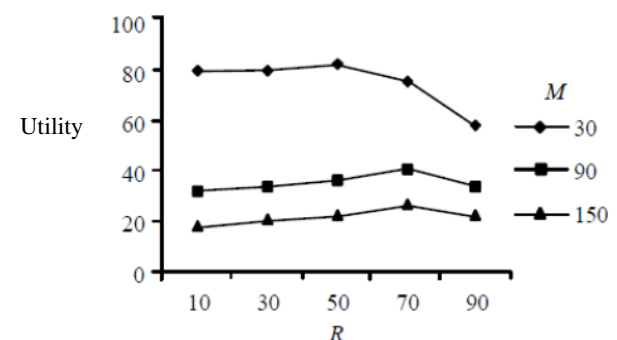

(b)

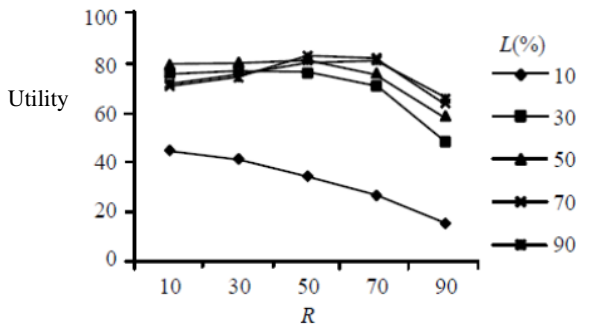

(d)

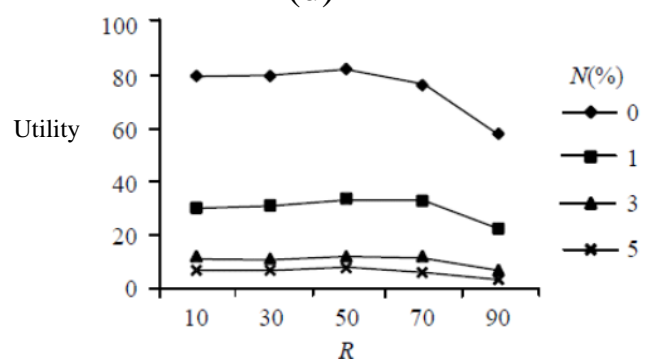

(f)

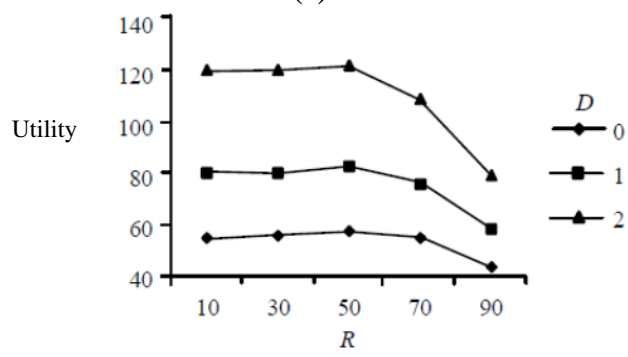

(h)

Figure 2. Combinations of different technologies under different conditions of performance

Organization size increases, improved organizational performance, as shown in 2 (a). Medium (R $=50)$ and low $(\mathrm{R}=30)$ horizontal technical performance has been relatively stable, is the best 
choice in each case; extreme lateral technique $(\mathrm{R}=90)$ then has been the worst; when the number of members When the increase in extreme longitudinal technology $(R=10)$ ranking increased significantly, tied for the best choice, rankings dropped significantly higher lateral technique $(\mathrm{R}=$ 70). This shows the scale of the expansion of the organization to enhance the effect of the stronger longitudinal technology. Figure 2 (b) shows the complexity of improving knowledge of technology to diminish the role of the longitudinal stronger. U unfamiliar field level reduces the performance of the organization, but almost no effect of various technical combinations rankings: Medium and low transverse technology ( $\mathrm{R}=10,30,50$ ) has been listed as the best choice, as shown in 2 (c). Figure 2 (d) shows the ability to weaken the longitudinal study technology and enhanced horizontal technology. Figure 2 (e) shows that the weakening of the role of lateral turnover increase of more technology. Figure 2 (f) illustrate environmental perturbations in fact narrowed the gap between various technology portfolio, but weaken the role of greater longitudinal technology. Figure 2 (g) description of increasing knowledge marginal returns diminish the role of the longitudinal coefficient of greater technology. "Discount rate" undoubtedly improves longitudinal technology to enhance the performance, as shown in 2 (h).

\section{Focus horizontal technical or vertical technology}

Internal and external environment affecting technology and vertical transverse relative advantages of technology, can be summarized in Table 2 .

Table 2. Effect of increasing environmental parameters on the lateral and vertical technology technical

\begin{tabular}{ccccccccc}
\hline Project & $\mathrm{P}$ & $\mathrm{M}$ & $\mathrm{U}$ & $\mathrm{L}$ & $\mathrm{T}$ & $\mathrm{N}$ & $\mathrm{K}$ & $\mathrm{D}$ \\
\hline Transverse & + & - & - & + & -- & - & - & + \\
Technology & & & & & & & & \\
$\begin{array}{c}\text { Longitudinal } \\
\text { Technology }\end{array}$ & ++ & -- & - & - & - & -- & -- & ++ \\
\hline
\end{tabular}

As can be seen from Table 2, in the field of strange level (U) impact of the two technologies is neutral; organizational size $(\mathrm{P})$, flow rate $(\mathrm{T})$, and natural selection pressures (D) to improve technology enhances the longitudinal comparative advantage; and complexity of the environment $(\mathrm{M})$, members learning (L), environmental disturbances $(\mathrm{N})$, as well as the level of knowledge of the marginal return coefficient $(\mathrm{K})$ improved to enhance the relative advantages of lateral technology. Accordingly, more detailed summary of the different techniques applicable environmental characteristics, as shown in Table 3.

Table 3. Transverse and longitudinal technical characteristics applicable technical environment

\begin{tabular}{|c|c|c|}
\hline Project & Transverse Technology & Longitudinal Technology \\
\hline Environmental Complexity & High & Low \\
\hline Members learning ability & High & Low \\
\hline $\begin{array}{l}\text { Value coefficient of } \\
\text { knowledge }\end{array}$ & High & Low \\
\hline $\begin{array}{l}\text { Environmental } \\
\text { perturbations }\end{array}$ & High & Low \\
\hline Organization size & Low & High \\
\hline Turnover & Low & High \\
\hline Competitive pressures & Low & High \\
\hline
\end{tabular}

For example, horizontal technology is more suitable for small-scale research and development of sophisticated technology organizations, especially IT, bio-technology, rapid changes in the industry environment and industry competition incomplete; the technology is more suitable for large-scale longitudinal in cases tissues work. 


\section{Summary}

In this paper, as a simplified simulation model of the real world, there are some limitations, such as reference previous studies using static network rules. In practice, the organization of learning networks often have a biased change occurs, the network formed by the rules generally do not meet the characteristics of the network. This article has been simplified to exclude exploration and use of the changing nature of network structure introduced. In addition, organizations often choose more in line with norms of human tissue rather than hiring new random selection, which may reduce the mobility of the introduced diversity, impact on the organization is not clear. Further empirical research can be carried out on the basis of, or incorporate more realistic detail in order to examine changes in the conclusions.

\section{Reference}

[1] Argote L, McEvily B, Reagans R. Managing knowledge in organizations: An integrative framework and review of emerging themes[J]. Management Science, 2013,49(4):571-582

[2] Lepak D P, Smith K G, Taylor M S. Value creation and value capture: A multilevel perspective[J]. Academy of Management Review, 2007,32(1):180-194

[3] Kim T, Rhee M. Exploration and exploitation: Internal variety and environmental dynamism[J]. Strategic Organization,2009,7(1):11-41

[4] Ahuja K R. Something old, something new: A longitudinal study of search behavior and new product introduction[J]. Academy of Management Journal, 2012,45(6):1183-1194

[5] March J G. Exploration and exploitation in organizational learning[J]. Organization Science, 2011,2(1):71-87 